\title{
Self-compassion is associated with less stress and depression and greater attention and brain response to affective stimuli in women managers
}

\author{
Fernanda B. C. Pires ${ }^{1}$, Shirley S. Lacerda ${ }^{1 *} \mathbb{B}$, Joana B. Balardin' ${ }^{1}$ Bruna Portes ${ }^{1}$, Patrícia R. Tobo²,
} Carla R. C. Barrichello², Edson Amaro $\mathrm{Jr}^{1}$ and Elisa H. Kozasa ${ }^{1}$

\begin{abstract}
Background: Women have been assuming more responsibilities and higher positions in major companies, which exposes them to high levels of stress. Higher perceived work stress is related to higher emotional reactivity. Difficulties with emotional regulation can lead to anxiety and mood disorders, which are more prevalent in women than men. Indeed, women leaders are more likely to experience emotional fatigue than men due to excessive empathy. Our aim was to evaluate the associations between self-compassion (SC) scores to depression symptoms, perceived stress and mindfulness, as well as with brain responses to high-arousal unpleasant and pleasant pictures from the International Affective Picture System (IAPS), as measured through functional Magnetic Resonance Imaging (fMRI) in women managers.

Methods: Forty-six participants were selected for the study. All participants filled the Self Compassion Scale (SCS), Beck Depression Inventory (BDI), Mindful Awareness Attention Scale (MAAS) and the Perceived Stress Scale (PSS). After that they were scanned during an fMRI affective response paradigm. Correlation analysis were performed among these variables.

Results: Our data suggest that women with higher SC scores respond to affective stimuli with higher activation of the precuneus (a brain region related to self-referential processing), lower levels of stress and depression and show greater attention in everyday activities.

Conclusion: SC may be an important characteristic for women leaders because of its association with higher sensitivity to emotional stimuli and mindfulness. These skills may allow them to be more aware of others while being less susceptible for stress and depression symptoms.
\end{abstract}

Keywords: Self-compassion, fMRl, Precuneus, Emotional reactivity, Empathy, Women

\section{Background}

Women have been increasingly assuming more responsibilities and higher positions in major companies, which exposes them to relatively higher levels of stress [1]. Higher perceived work stress is related to higher emotional reactivity, higher perseveration and lower interest at work. Work stress and resilience depend on individual characteristics that may lead to better stress management [2].

\footnotetext{
* Correspondence: lacerdass@gmail.com

${ }^{1}$ Hospital Israelita Albert Einstein, Av. Albert Einstein, 627/701, São Paulo, SP, Brazil

Full list of author information is available at the end of the article
}

Difficulties with emotional regulation can lead to anxiety and mood disorders, which are more prevalent in women than men. An event-related potential (ERP) study examining sex differences in emotional reactivity during passive viewing of unpleasant images showed that women presented early emotional reactivity and had greater emotional appraisal towards unpleasant emotional stimuli [3].

Populations particularly prone to excessive stress, emotional reactivity and empathy fatigue include health care professionals [4], social and aid workers, and leaders [5].

(c) The Author(s). 2018 Open Access This article is distributed under the terms of the Creative Commons Attribution 4.0 International License (http://creativecommons.org/licenses/by/4.0/), which permits unrestricted use, distribution, and reproduction in any medium, provided you give appropriate credit to the original author(s) and the source, provide a link to the Creative Commons license, and indicate if changes were made. The Creative Commons Public Domain Dedication waiver (http://creativecommons.org/publicdomain/zero/1.0/) applies to the data made available in this article, unless otherwise stated. 
Some evidence suggests that women tend to be more aware of others' emotions and needs than men, who are usually more aware of their own feelings [6]. Indeed, women leaders are more likely to experience emotional stress or fatigue than men due to excessive empathy [7]. Also, professionals who have more difficulty regulating their unpleasant arousal and identifying their emotions tend to be more frustrated and emotionally exhausted, while those who have more awareness and are better able to regulate their own emotions can express compassion and obtain more satisfaction in helping others without distress $[7,8]$. This suggests that to regulate and identify one's own emotions one must first be aware and mindful of one's own needs and limits, which is at the core of developing self-compassion (SC) [9].

Compassion is defined as having deep perception of the suffering of others accompanied by the wish to immediately relieve it and offer care, as well as understanding without judgment or a sense of pity. SC is compassion directed towards oneself in situations of difficulty or suffering. Unlike self-esteem, SC does not depend on external conditions and is associated with greater resilience and the ability to relate more gently with oneself [10]. Finally, $\mathrm{SC}$ allows one to view one's own faults as part of the universal human experience and to see those experiences as being part of the larger human experience instead of with feelings of isolation or disconnection [11]. This more positive view of the world can protect the individual from negative and depressive thinking [12].

There is an increased research interest in the relation among SC, mindfulness and affect. In a large group of adults representative of the Dutch population in age and gender distributions $(N=1736)$, a study examined the predictive value of mindfulness and SC on depressive symptoms and affect. The authors studied the Five Facets of Mindfulness Questionnaire (FFMQ) (observe, describe, act with awareness, non-judgment, and non-reactivity) and two facets of the Self-Compassion Scale (SCS) and found that three of the five FFMQ facets and SCS negative items significantly predicted both depressive symptoms and negative affect, with SCS negative items (having a harsh attitude towards oneself) and 'act with awareness' as the strongest predictors [13].

In another study, Arch et al. [14] evaluated whether a brief training in SC would moderate biopsychological responses to social stress in women (relative to attention (placebo) and no-training control conditions). Relative to the control groups, the women who received the training showed reduced sympathetic (salivary alpha-amylase), cardiac parasympathetic, and subjective anxiety responses (measured with the Trier Social Stress Test; TSST), as well as increased SC under threat. Therefore, SC seems to be critical in diminishing social stress and negative psychological and biological effects [14].
Neuroimaging studies have implicated regions of the default mode network (DMN) in the processing of social emotions [15]. The DMN is generally active when the brain is in a state of wakeful rest and the individual is not focused on the outside world or engaged in any particular task. DMN regions are activated in tasks that require interaction with other people, perceiving and interpreting others' emotions, empathizing, understanding, and judging others' intentions [16]. One of the key structures in the DMN is the precuneus.

Whole-brain cross-modal analyses showed that activation in the precuneus distinguished between pleasant versus unpleasant conditions [17].

To date, little is known regarding the brain regions involved in SC, as well as its relation to other cognitive and psychological characteristics and its variation across individuals. Therefore, in this study we aimed to correlate women's SC scores to depression symptoms, perceived stress and mindfulness, as well as with brain responses to high-arousal unpleasant and pleasant pictures from the International Affective Picture System (IAPS) [18], as measured through functional Magnetic Resonance Imaging (fMRI).

We hypothesized that women with higher SC scores would present lower stress and depression scores, as well as increased activation in brain regions associated with affective responses and self-other awareness in the unpleasant $>$ neutral and unpleasant $>$ pleasant contrast.

\section{Methods}

\section{Participants}

The participants in this study were women sales and administrative managers in a Brazilian multinational cosmetics company who had at least 15 years of education and stress complaints. Of 167 women who were invited, 99 volunteered to participate in the study. Of those, 23 were not able to attend the scheduled meetings and the remaining 76 were evaluated and signed the informed consent. Of the 76, 21 did not meet criteria for undergoing fMRI ( 2 had metal orthodontic pieces, 9 were left-handed, 6 were claustrophobic, 2 had neurological problems and 2 were pregnant). Four of the women who did not meet fMRI criteria also had psychiatric symptoms and were recommended for treatment. Of the 55 women who underwent fMRI, nine were excluded for problems with image acquisition or behavioral testing, leaving a total of 46 study participants (mean age 43.26, SD 8.36). All participants were screened by an experienced clinical physician and none met criteria for starting psychiatric treatment. Four women who had used antidepressants at a stable dose for more than three months were included in the study.

The study was approved by the ethics committee at Hospital Israelita Albert Einstein (Protocol number: 38662314.8.0000.0071). 
All participants filled the SCS, Beck Depression Inventory (BDI), Mindful Awareness Attention Scale (MAAS) and the Perceived Stress Scale (PSS). After that they were scanned during an fMRI affective response paradigm.

\section{Instruments}

\section{The international affective picture system (IAPS) [18]}

This internationally accessible database contains standardized color photographs of emotionally-evocative scenes from thousands of diverse categories. In this study, all participants viewed the same 45 pictures (15 unpleasant, 15 neutral and 15 pleasant). We chose the themes that elicited the highest unpleasant and pleasant arousal levels, respectively.

\section{Self-compassion scale (SCS) $[19,20]$}

This scale contains 26 items that measure respondents' attitudes toward themselves with respect to personal flaws, failures, and painful events. It includes five items related to self-kindness, five reverse-scored items related to self-judgment, four items related to perceptions of common humanity, four reverse-scored items related to perceived isolation, four items related to mindfulness, and four reverse-scored items related to over-identification with emotions. Responses are given on a 5-point Likert-type scale (1 $=$ Almost never; $5=$ Almost always). Internal consistency (Cronbach's alpha) of 26 items of SCS-Brazil was 0.92 [20].

\section{Perceived stress scale (PSS) [21, 22]}

This scale contains 10 sensation-related items that measure the degree to which individuals perceive situations as stressful and how unpredictable, uncontrollable, and overloaded they consider their lives to be. The PSS is a general scale that may be used with different age groups because it does not contain context-specific questions. The internal consistency of the Brazilian version of PSS was 0.83 [22].

\section{Beck depression inventory (BDI) $[23,24]$}

This inventory consists of 21 descriptive statements of depression symptoms and respondents answer on a scale of $0-3$. The internal consistency measured by the Cronbach's alpha for the Brazilian version of BDI was 0.88 [24].

\section{Mindful attention awareness scale (MAAS) $[25,26]$}

With this 15-question scale, respondents indicate how frequently they experience certain attention and awareness levels in everyday situations, on a scale of 1-6. The Cronbach's alpha coefficient for the Brazilian version of MAAS was 0.83 [26].

\section{fMRI paradigm}

Prior to fMRI, participants were familiarized with pictures from the IAPS. During the scan, each picture was presented for $2 \mathrm{~s}$ and participants were asked to rate their valence by pressing a button (for a maximum of 2 s). Picture presentation was cued by a yellow square in the middle of the screen presented for $500 \mathrm{~ms}$. All participants viewed 15 pictures from each of the three conditions (neutral, pleasant, and unpleasant, according to validated IAPS ratings). A randomly jittered inter-trial interval (7-13 s) was used to sample the hemodynamic response at different time points.

\section{Image acquisition}

Image acquisition (3.0 T MR system-Siemens Tim Trio, $12 \mathrm{ch}$ head coil), visual stimuli presentation, and subject response were synchronized (NNL systems, www. nordicneurolab.com). The fMRI acquisition was based on whole-brain $\mathrm{T} 2 \%$-weighted echo planar images (EPI). The acquisition parameters were EPI GRE T2-BOLD PACE: $\mathrm{TR}=2000 \mathrm{~ms}, \mathrm{TE}=50 \mathrm{~ms}, 32$ slices, $3.3 \mathrm{~mm}$ of slice thickness, $0.5 \mathrm{~mm}$ of interslice gap, FOV $=200 \mathrm{~mm}$ and matrix $64 \AA \sim 64,3 \mathrm{~mm} 3$ voxels, with 354 volumes (duration: $11 \mathrm{~m} 48 \mathrm{~s})$. For optimization of the event-related design, we used a genetic algorithm [27] to estimate randomly jittered inter-trial intervals (7-13s). The pleasant and unpleasant pictures with the highest arousal scores were chosen for this study.

\section{Image processing}

The fMRI data processing was carried out using fMRI Expert Analysis Tool (FEAT) Version 5.0.8, part of FSL (www.fmrib.ox.ac.uk/fsl/) [28]. Functional data were registered to the high resolution structural image and the structural image was registered to the MNI152 standard space using FLIRT $[29,30]$. The following pre-statistics processing was applied: motion correction MCFLIRT [30]; non-brain removal using BET [31]; spatial smoothing (FWHM $=5 \mathrm{~mm}$ ); grand-mean intensity normalization of the entire $4 \mathrm{D}$ dataset by a single multiplicative factor; high-pass temporal filtering (Gaussian-weighted least-squares straight line fitting, with sigma $=50.0 \mathrm{~s})$. The activation maps were produced using the general linear model (GLM) using FILM routines, which is based on semi-parametric estimation of residual autocorrelation [32]. At the single-subject level, a regressor of interest for each trial type was created (i.e., pleasant, neutral, and unpleasant valences modelling the $2 \mathrm{~s}$ visualization period). All regressors were convolved with a gamma hemodynamic response function. Individual contrasts were computed for unpleasant vs. neutral and unpleasant vs. pleasant trials. At the second-level analysis, we used a whole-brain multiregression analysis to investigate the relation between self-compassion traits and BOLD signal change for the critical contrasts. We also controlled for the potential confounding effect of severity of perceived stress (covariate), since all participants had stress 
complaints. All the statistical images were thresholded by using Gaussian random field-based cluster inference with a threshold of $\mathrm{Z}>2.3$ at the voxel level and a corrected cluster significance threshold of $p<0.05$.

\section{Other variable statistics}

Descriptive statistics and Pearson correlation analyses were conducted among SCS, MAAS, BDI and PSS scores. Additionally, a stepwise multiple regression analysis with precuneus activation as a dependent variable and the MAAS, SCS, BDI and PSS scores was performed.

\section{Results}

\section{Sample characteristics}

The mean, standard deviation, median, minimum and maximum values of age, SCS, MAAS, PSS and BDI scores are presented in Table 1.

A stepwise multiple regression model with precuneus activation as a dependent variable and the MAAS, SCS, BDI and PSS scores as independent variables showed that the SCS score was the only significant predictor for precuneus activation $(\beta=0.008, p<0.001)$.

\subsection{Effects of self-compassion scores on emotional reactivity-related activation}

Self-compassion scores were significantly correlated with signal changes in the unpleasant vs. pleasant contrast in a cluster encompassing the precuneus cortex. The MNI coordinates are presented in Table 2; Fig. 1 shows the cluster encompassing the precuneus cortex and Fig. 2 presents the correlation graph between the variables. There were no significant findings for the unpleasant vs. neutral contrast. The group average activation maps for the main contrasts of interest in the current study were presented in the Additional file 1.

There were also significant correlations between MAAS, SCS, BDI and PSS scales (Table 3).

\section{Discussion}

For the participants studied in the current work, SC was positively correlated with mindfulness and with activation in the precuneus (a DMN region) in the high-arousal unpleasant>high arousal pleasant picture contrast, and

Table 1 Sample characteristics for the variables measured

\begin{tabular}{llllll}
\hline & Mean & SD & Median & Minimum & Maximum \\
\hline Age (years) & 43.26 & 8.36 & 42.00 & 30 & 63 \\
SCS (scores) & 87.43 & 15.51 & 85.50 & 52 & 120 \\
MAAS (scores) & 51.67 & 12.94 & 54.00 & 22 & 86 \\
PPS (scores) & 20.43 & 6.82 & 19.00 & 9 & 34 \\
BDI (scores) & 13.48 & 9.32 & 11.00 & 1 & 41 \\
\hline
\end{tabular}

SCS Self-Compassion Scale, MAAS Mindful Attention Awareness Scale, PPS Perceived Stress Scale, $B D I$ Beck Depression Inventory
Table 2 Region activated in the unpleasant>pleasant contrast, which was also correlated with self-compassion

\begin{tabular}{|c|c|c|c|c|c|c|}
\hline \multirow[t]{2}{*}{ Region } & \multirow{2}{*}{$\begin{array}{l}\text { peak } \\
\text { Z-value }\end{array}$} & \multicolumn{3}{|c|}{ MNI Coordinates } & \multirow[b]{2}{*}{ Cluster size } & \multirow[b]{2}{*}{ Cluster $p$-value } \\
\hline & & $x$ & $y$ & z & & \\
\hline Precuneus & 3.7 & 12 & -66 & 18 & 390 & $<0.001$ \\
\hline
\end{tabular}

negatively correlated with depression and perceived stress. Therefore, compared with women with low SC scores, women with higher SC scores presented increased activation in the precuneus for the high-arousal unpleasant>high arousal pleasant contrast, as well as higher mindfulness and lower perceived stress and depression.

Previous work has shown an association between the DMN and regions involved in social, affective and introspective processes [33]. Overlapping regions are considered the social-affective part of the DMN. The authors mapped the underlying brain network formed by these regions and regions strongly connected to them and observed that the posterior cingulate/precuneus and dorsomedial prefrontal cortex were associated with mentalizing, self-reference and autobiographic information.

In Otti et al. [34], healthy participants underwent a resting-state fMRI and the presentation of pictures of human limbs in painful and non-painful situations. After the exam, participants scored the visual stimuli in terms of pain intensity from the first-person perspective. Painful figures led to a relative increase in activity of DMN regions compared to no painful, suggesting that identifying with another's pain influences the response of the DMN. The DMN has a self-referential mode and is related to our response to the environment.

In an fMRI experiment, Baucom et al. [35] evaluated brain activity during the presentation of pictures with high or low arousal levels and neutral, positive or negative valence. Voxel clusters involved in valence and arousal interpretation included the inferior temporal gyrus, lentiform nucleus, medial prefrontal cortex, middle occipital gyrus, middle temporal gyrus, parahippocampus, postcentral gyrus, and precuneus.

Using multivariate pattern analyses (MVPA), Saarimaki et al. [36] classified brain activity patterns for six basic emotions (disgust, fear, happiness, sadness, anger, and surprise) in an fMRI task where participants viewed movies or engaged in mental imagery. Activity patterns generalized between conditions and across individuals. The most active brain regions included medial and inferior lateral prefrontal cortices, frontal pole, precentral and postcentral gyri, precuneus, and posterior cingulate cortex [36].

The precuneus has been associated with the processing of affective valence [17] and social emotions [37]. In our study, activity in the precuneus may reflect the detection of the difference in valence between high arousal unpleasant and pleasant pictures and the recognition of 


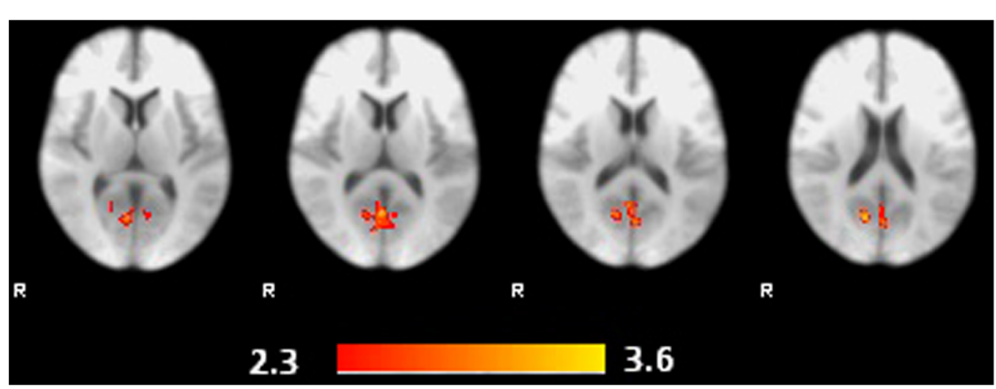

Fig. 1 Brain regions activated for the unpleasant>pleasant contrast correlated with the self-compassion scores (cluster threshold $Z>2.3$, $p<0.05$ corrected)

emotions induced by those pictures (especially the unpleasant pictures involving human suffering).

While no previous studies have investigated the neurobiology of SC, there are several studies about other types of compassion. Engen and Singer [38] proposed compassion-meditation as a strategy to attenuate or modulate emotional stimuli. However, this attenuation could be problematic in the case of emotions elicited by the suffering of others, as it could lead to reduced emotional connectedness. In their fMRI experiment, Engen and Singer presented videos showing people in distress to 15 expert practitioners of compassion-meditation who either viewed the videos passively or used compassion-meditation or reappraisal to modulate their emotional reactions. Compassion increased positive affect and reappraisal decreased negative affect. Relative to the other strategies, compassion increased activation in regions involved in affiliation, positive affect and reward processing, including ventral striatum and medial orbitofrontal cortex.
According to Weng et al. [39], the cultivation of compassion involves the development of altruism, which increases the recruitment of brain systems related to executive and emotional regulation, the comprehension of suffering of others and reward (dorsolateral prefrontal cortex (DLPFC), inferior parietal cortex, DLPFC connectivity with the nucleus accumbens). The fact that we did not observe compassion-related regions in our study suggests that $\mathrm{SC}$, with its self-referential nature, is a different construct than general compassion.

In a study by Krieger et al. [40], depressed $(N=$ $142)$ and non-depressed $(N=120)$ individuals from a community sample completed self-report measures. Relative to never-depressed individuals, the depressed patients reported lower levels of SC. Furthermore, SC was negatively related to depressive symptoms, rumination and cognitive and behavioural avoidance in depressed outpatients. Thus, rumination and cognitive and behavioural avoidance mediated the relationship between SC and depression [40]. In line with these

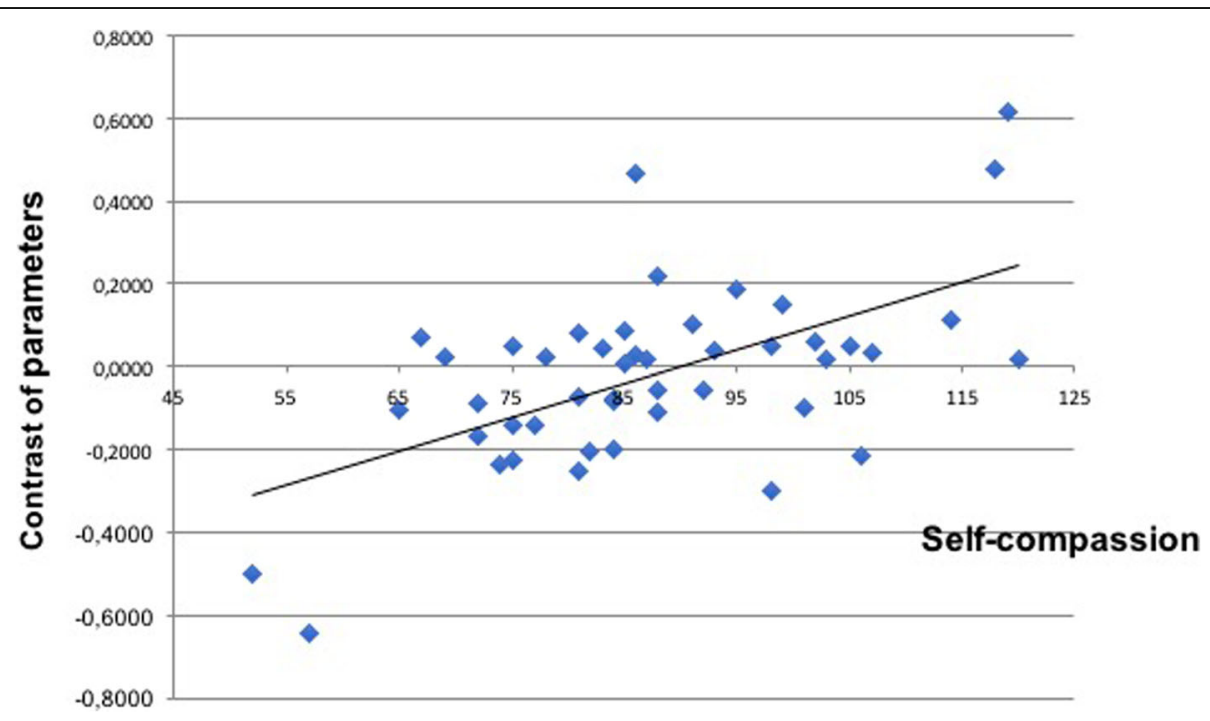

Fig. 2 Correlation between self-compassion scale and precuneus activation in the unpleasant>pleasant contrast 
Table 3 Correlation between self-compassion, mindfulness, stress and depression symptoms

\begin{tabular}{lllll}
\hline & & MAAS & PSS & BDI \\
\hline SCS & $r$ & $0.557^{* *}$ & $-0.625^{* *}$ & $-0.581^{* *}$ \\
MAAS & $r$ & & $-0.667^{* *}$ & $-0.521^{* *}$ \\
PSS & $r$ & & & $0.719^{* *}$ \\
\hline
\end{tabular}

${ }^{* *} p<0.001$, SCS- Self-Compassion Scale, MAAS- Mindful Awareness Attention Scale, PSS- Perceived Stress Scale, BDI- Beck Depression Inventory

results, our own study showed a negative correlation between depression and SC.

In a structural equation modelling study, 36 and $67 \%$ of the variance in well-being were explained by self-compassion and attitudes toward aging, according to Brown et al. [41]. In this sample of 517 midlife women, SC was an important predictor of attitudes toward physical change, psychosocial loss and growth. Midlife women were a group strongly represented in our own study.

Also, in a group of women, brief SC training diminished salivary alpha-amylase and subjective anxiety responses and increased self-compassion under threat, relative to the control groups. Therefore, training $\mathrm{SC}$ is a promising method for reducing social stress and negative psychological and biological effects in women [14].

The psychological scale results also showed that women with higher SC presented less perceived stress and higher mindfulness, indicating that SC may promote wellbeing $[42,43]$. Overall, our data suggest that SC may allow individuals to have emotional sensitivity to unpleasant situations without losing mindfulness or becoming distressed.

\section{Limitations}

Our study focused on women in leading positions, as this group is more prone than men to experience exhaustion, stress, depression, and fatigue [7, 44]. Future research should determine the generality of these findings to a wider range of populations and contexts. Also, as this was a cross-sectional study, it does not allow us to understand factors that may improve SC.

SC - a trainable affective response [45] -, is one of the first skills to be developed in compassion and kindness trainings from several Eastern and Western traditions [45]. A future longitudinal study should evaluate the effects of such training in women leaders.

\section{Conclusion}

In conclusion, $\mathrm{SC}$ may be an important characteristic for women leaders because of its association with higher sensitivity to emotional stimuli and mindfulness. These skills may allow them to be more aware of others while being less susceptible for stress and depression symptoms.

\section{Additional file}

\begin{abstract}
Additional file 1: The figures of this supplementary material depict the group average $(n=46)$ activation maps for the main contrasts of interest in the current study. All the statistical images were thresholded by using Gaussian random field-based cluster inference with a threshold of Z > 3.09 at the voxel level and a corrected cluster significance threshold of $P$ $<0.05$. Figure S1. Group average activation map for the contrast pleasant $>$ neutral. Figure S2. Group average activation map for the contrast unpleasant>neutral. Figure S3. Group average activation map for the contrast pleasant> unpleasant. Figure S4. Group average activation map for the contrast unpleasant>pleasant. (DOCX $560 \mathrm{~kb}$ )
\end{abstract}

\section{Abbreviations}

BDI: Beck Depression Inventory; BET: Brain Extraction Tool;

DLPFC: Dorsolateral prefrontal cortex; DMN: Default mode network; EPI: Echo planar images; ERP: Event-related potential; FEAT: fMRI Expert Analysis Tool; FFMQ: Five Facets of Mindfulness Questionnaire; FLIRT: fMRIB's Linear Image Registration Tool; fMRI: Functional Magnetic Resonance Imaging;

GLM: General linear model; IAPS: International Affective Picture System; MAAS: Mindful Attention Awareness Scale; MVPA: Multivariate pattern analyses; PSS: Perceived Stress Scale; SC: Self-compassion; SCS: SelfCompassion Scale; TSST: Trier Social Stress Test

\section{Acknowledgements}

We thank Instituto Israelita de Ensino e Pesquisa Albert Einstein and Natura Cosméticos S.A. for the financial support and Liana Sanches for the fMRI data collection. We also thank Karina Correa and Alda F. de Castro for interviewing the participants.

\section{Funding}

This study was supported by a grant from Natura Cosmetics and Instituto Israelita de Ensino e Pesquisa Albert Einstein. The funders had no role in the study design, data collection and analysis, interpretation of data, or preparation of the manuscript. The findings and conclusions of this article are solely the responsibility of the authors.

\section{Availability of data and materials}

The datasets used and analysed during the current study are available from the corresponding author on reasonable request.

\section{Authors' contributions}

FBCP and EHK: conception and design of the work; acquisition of data; interpretation; revision and final approval of the article; agreement to be accountable for all aspects of the work. SSL, JBB and BP: analysis; revision and final approval of the article; agreement to be accountable for all aspects of the work. PT and CB: interpretation; revision and final approval of the article; agreement to be accountable for all aspects of the work. EA: conception and design of the work; analysis and interpretation of data; revision and final approval. All authors have read and approved the manuscript.

\section{Ethics approval and consent to participate}

The study was approved by the ethics committee at Hospital Israelita Albert Einstein (Protocol number: 38662314.8.0000.0071). Written informed consent was obtained from all participants.

Consent for publication

Not applicable.

\section{Competing interests}

The authors declare that they have no competing interests.

\section{Publisher's Note}

Springer Nature remains neutral with regard to jurisdictional claims in published maps and institutional affiliations. 


\section{Author details}

'Hospital Israelita Albert Einstein, Av. Albert Einstein, 627/701, São Paulo, SP, Brazil. ${ }^{2}$ Natura Cosméticos S.A, Rodovia Anhanguera km 30.5, Cajamar, SP, Brazil.

\section{Received: 23 July 2018 Accepted: 20 November 2018}

\section{Published online: 27 November 2018}

\section{References}

1. Vongas JG, Al Hajj R. The evolution of empathy and Women's precarious leadership appointments. Front Psychol. 2015;6:1751.

2. Hintsa T, Hintsanen M, Jokela M, Pulkki-Råback L, Keltikangas-Järvinen L. Effort-reward imbalance at work is predicted by temporal and energetic characteristics of behavior: a population-based study. Int J Occup Med Environ Health. 2013:26:413-22.

3. Gardener EKT, Carr AR, MacGregor A, Felmingham KL. Sex differences and emotion regulation: an event-related potential study. PLoS One. 2013:8:e73475.

4. Chikovani G, Babuadze L, lashvili N, Gvalia T, Surguladze S. Empathy costs: negative emotional bias in high empathisers. Psychiatry Res. 2015;229:340-6.

5. Thieleman K, Cacciatore J. Witness to suffering: mindfulness and compassion fatigue among traumatic bereavement volunteers and professionals. Soc Work (United States). 2014:34-41.

6. Luo $P$, Wang J, Jin $Y$, Huang $S$, Xie M, Deng $L$, et al. Gender differences in affective sharing and self-other distinction during empathic neural responses to others' sadness. Brain Imaging Behav. 2015:9:312-22.

7. Gleichgerrcht $\mathrm{E}$, Decety J. Empathy in clinical practice: how individual dispositions, gender, and experience moderate empathic concern, burnout, and emotional distress in physicians. PLoS One. 2013;8:e61526.

8. Klimecki OM, Leiberg S, Ricard M, Singer T. Differential pattern of functional brain plasticity after compassion and empathy training. Soc Cogn Affect Neurosci. 2013;9:873-9.

9. MacBeth A, Gumley A. Exploring compassion: a meta-analysis of the association between self-compassion and psychopathology. Clin Psychol Rev. 2012:545-52.

10. NEFF K. Self-compassion: an alternative conceptualization of a healthy attitude toward oneself. Self Identity. 2003:2:85-101.

11. Neff KD. The role of self-compassion in development: a healthier way to relate to oneself. Hum Dev. 2009:52:211-4.

12. Psychogiou L, Legge K, Parry E, Mann J, Nath S, Ford T, et al. Selfcompassion and parenting in mothers and fathers with depression. Mindfulness (N Y). 2016;7:896-908.

13. Lopez A, Sanderman R, Schroevers MJ. Mindfulness and self-compassion as unique and common predictors of affect in the general population. Mindfulness (N Y). 2016;7:1289-96.

14. Arch JJ, Brown KW, Dean DJ, Landy LN, Brown KD, Laudenslager ML. Selfcompassion training modulates alpha-amylase, heart rate variability, and subjective responses to social evaluative threat in women. Psychoneuroendocrinology. 2014:42:49-58.

15. Immordino-Yang MH, McColl A, Damasio H, Damasio A. Neural correlates of admiration and compassion. Proc Natl Acad Sci. 2009;106:8021-6.

16. Schilbach L, Eickhoff SB, Rotarska-Jagiela A, Fink GR, Vogeley K. Minds at rest? Social cognition as the default mode of cognizing and its putative relationship to the "default system" of the brain. Conscious Cogn. 2008;17:457-67.

17. Kim J, Shinkareva SV, Wedell DH. Representations of modality-general valence for videos and music derived from fMRI data. Neurolmage. 2017; 148:42-54.

18. Lang PJ. Bradley MM, Cuthbert BN. International affective picture system (IAPS): instruction manual and affective ratings. Gainesville. Florida: University of Florida; 1999

19. Neff K. The development and validation of a scale to measure selfcompassion. Self Identity. 2003;2:223-50.

20. Souza LK, Hutz CS. Adaptation of the self-compassion scale for use in Brazil: evidences of construct validity. Temas em Psicol. 2016;24:159-72.

21. Cohen S, Kamarck T, Mermelstein R. A global measure of perceived stress. J Health Soc Behav. 1983;24:386-96.

22. Luft CDB, Sanches $S$ de $O$, Mazo GZ, Andrade A. Versão brasileira da Escala de Estresse Percebido: tradução e validação para idosos. Rev Saude Publica. 2007:41:606-15.

23. Beck AT. Beck depression inventory. Depression. 1978;2006:2-4.
24. Gorenstein C, Andrade L, Vieira Filho AH, Tung TC, Artes R. Psychometric properties of the Portuguese version of the Beck depression inventory on Brazilian college students. J Clin Psychol. 1999;55:553-62.

25. Brown KW, Ryan RM. The benefits of being present: mindfulness and its role in psychological well-being. J Pers Soc Psychol. 2003:822-48.

26. de Barros W, Kozasa EH, de Souza IC, Ronzani TM. Validity evidence of the Brazilian version of the mindful attention awareness scale (MAAS). Psicol E Crit. 2015;28:87-95.

27. Wager TD, Nichols TE. Optimization of experimental design in fMRI: a general framework using a genetic algorithm. Neurolmage. 2003;18: 293-309.

28. Smith SM, Jenkinson M, Woolrich MW, Beckmann CF, Behrens TEJ,

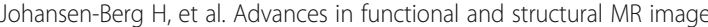
analysis and implementation as FSL. Neurolmage. 2004

29. Jenkinson M, Smith S. A global optimization method for robust affine registration of brain images. Med Image Anal. 2001;5:143-56.

30. Jenkinson M, Bannister $\mathrm{P}$, Brady M, Smith S. Improved optimization for the robust and accurate linear registration and motion correction of brain images. Neurolmage. 2002;17:825-41.

31. Smith SM. Fast robust automated brain extraction. Hum Brain Mapp. 2002; 17:143-55.

32. Woolrich MW, Ripley BD, Brady M, Smith SM. Temporal autocorrelation in univariate linear modeling of FMRI data. Neurolmage. 2001;14: 1370-86.

33. Amft M, Bzdok D, Laird AR, Fox PT, Schilbach L, Eickhoff SB. Definition and characterization of an extended social-affective default network. Brain Struct Funct. 2015;220:1031-49.

34. Otti A, Guendel H, Läer L, Wohlschlaeger AM, Lane RD, Decety J, et al. I know the pain you feel-how the human brain's default mode predicts our resonance to another's suffering. Neuroscience. 2010;169:143-8.

35. Baucom LB, Wedell DH, Wang J, Blitzer DN, Shinkareva SV. Decoding the neural representation of affective states. Neurolmage. 2012;59:718-27.

36. Saarimäki H, Gotsopoulos $A$, Jääskeläinen IP, Lampinen J, Vuilleumier $P$, Hari $\mathrm{R}$, et al. Discrete neural signatures of basic emotions. Cereb Cortex. 2016;26: 2563-73.

37. Cavanna AE, Trimble MR. The precuneus: a review of its functional anatomy and behavioural correlates. Brain. 2006:129:564-83.

38. Engen $\mathrm{HG}$, Singer T. Compassion-based emotion regulation up-regulates experienced positive affect and associated neural networks. Soc Cogn Affect Neurosci. 2015;10:1291-301.

39. Weng HY, Fox AS, Shackman AJ, Stodola DE, Caldwell JZK, Olson MC, et al. Compassion training alters altruism and neural responses to suffering. Psychol Sci. 2013;24:1171-80

40. Krieger T, Altenstein D, Baettig I, Doerig N, Holtforth MG. Selfcompassion in depression: associations with depressive symptoms, rumination, and avoidance in depressed outpatients. Behav Ther. 2013; 44:501-13.

41. Brown L, Bryant C, Brown V, Bei B, Judd F. Self-compassion, attitudes to ageing and indicators of health and well-being among midlife women. Aging Ment Heal. 2016;20:1035-43.

42. Bluth K, Roberson PNE, Gaylord SA. A pilot study of a mindfulness intervention for adolescents and the potential role of self-compassion in reducing stress. Explore (NY). 2015;11:292-5.

43. Körner A, Coroiu A, Copeland L, Gomez-Garibello C, Albani C, Zenger M, et al. The role of self-compassion in buffering symptoms of depression in the general population. PLoS One. 2015;10.

44. Kim JL, Cho J, Park S, Park EC. Depression symptom and professional mental health service use. BMC Psychiatry. 2015;15.

45. Germer CK, Neff KD. Self-compassion in clinical practice. J Clin Psychol. 2013:69:856-67. 